\title{
Bending Virtual Spring-Damper: A Solution to Improve Local Platoon Control
}

\author{
Jean-Michel Contet, Franck Gechter, Pablo Gruer, \\ and Abderrafiaa Koukam \\ University of Technology of Belfort-Montbeliard (UTBM), \\ Systems and Transportation Laboratory (SET), Belfort, France \\ \{jean-michel.contet, franck. gechter, \\ pablo.gruer, abder.koukam\}@utbm.fr \\ http://set.utbm.fr/
}

\begin{abstract}
This article presents a local control approach to linear vehicle platooning. Linear platoon systems are sets of vehicles that use local or global perception capabilities to form a train configuration, without any hard grip element. Public transportation is beginning to interest in platoon systems as a technological base to conceive new services. The main problem related to platoon system's control corresponds with maintaining inter-vehicle distance. In literature, the platoon's geometry control problem is treated according to two approaches: global or local vehicle control. This paper focuses on a local approach which does not require sophisticated sensors and/or costly road equipment. This local control approach intends to obtain very good global matching to arbitrary trajectories, only from local perception which consists in measuring the vectorial distance between a given vehicle and its predecessor. The behavior of each platoon vehicle is determined from a physics inspired multi agent interaction model based on a virtual spring-damper. Furthermore, stability, platoon safety properties are checked using physics and mathematical proofs. Finally, simulation is used to measure trajectory error and inter-vehicle distance evolution.
\end{abstract}

Keywords: Platoon, local control, stability proof, simulation.

\section{Introduction}

Platoon systems can be defined as sets of vehicles that apply local or global perception capabilities to adopt and maintain a train-like or other geometric configuration or formation. Train platoon configurations are also called linear platoons. Control of vehicles' movement within a linear platoon can be decomposed into two main subproblems: longitudinal control and lateral control. Longitudinal control consists in controlling braking and acceleration in order to stabilize the distance between following vehicles. Lateral control consists in determining a vehicle's direction according to the platoon's trajectory. Linear platoon configurations (Cf. Fig. 1) attract considerable attention as an approach to innovative 


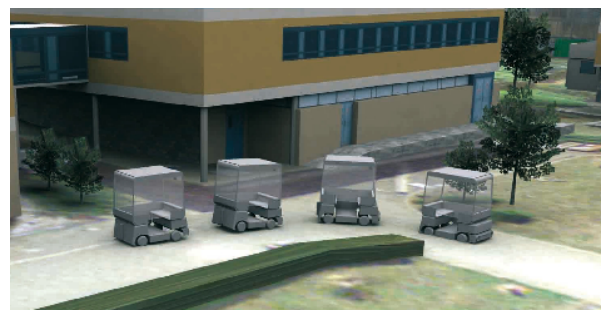

Fig. 1. Example of linear platoon simulation in front of System and Transportation laboratory (SET)

transportation systems [1. Their main impact [2], when applied to urban transportation, are: (i) reducing private car affluence (i.e. avoiding traffic jams), (ii) improving security thanks to automatic driving assistance (obstacle detection and avoidance, automatic car parking,...). Platoon based transportation systems are supposed to exhibit a set of convenient properties: adaptability to particular user's service requests, reconfigurability to accommodate a variable number of resources, depending on demand and others.

From the scientific and technological point of view, platoon system's control includes some relevant challenges. The main problem related to platoon systems relates to inter-vehicle distance control. In literature, the control of platoon's global geometry can be divided into two main approach: global or local vehicle control. Within the global approaches each following vehicle is made aware of the leading vehicle trajectory. This solution generally uses some positioning systems such as GPS 910]. According to the global control strategy, the leading vehicle broadcasts driving information (steering and speed) to each follower vehicle (i. e., the European Chauffeur project [11]). This approach yields very good trajectory following but the global position perception by GPS or other technology implies some road adaptation to avoid tunneling or canyon effects. Moreover, a safe, reliable communication network between vehicle is required. To concluded, the global control approach gives the best results with strong constraints on sensors (high cost), road adaptation and communication reliability between vehicles.

On the other hand, local control consists in computing the vehicle's command references (acceleration and direction) only from vehicle's own perceptions. Most of the lateral or longitudinal control strategies proposed within local approaches based on PID (Proportional, Integral, Derivative) controllers [121314] or other regulation-loop based algorithm [11/516 1723]. Some other works, propose to base on simulated physical phenomena to calculate vehicle's control references. For instance, Gehrig and Stein [18] take inspiration from physical particle forces. Soo-yeong $Y i$ and Kil-to Chong [19] model immaterial grip by means of an impedance control mechanism. The local approach has been criticized because of their relatively poor trajectory matching and the accordion effect they produce during platoon evolution. Indeed, naive local control approaches increase the trajectory matching error since a follower vehicle tends to anticipate the direction change. On the other hand the local approach, unlike the global one, 
does not require any sophisticated and highly fallible sensor and can be used in any environment, without any road upgrading.

The paper proposes a local approach which improves the trajectory in any curved trajectory. This approach is based on a virtual spring-damper which bends to avoid curve anticipation. This paper is structured as follows. The Formal (behavior and interaction) Models are presented. Then, stability proofs are presented using mathematical and physical points of view. Finally simulation results are shown. This paper concludes by stressing main contributions and by drawing some perspectives and future works.

\section{Formal Models}

\subsection{Behavioral Model}

In the approach presented here, an alternative to centralized control, vehicles are autonomous entities in mutual interaction. Consequently, the intended embodiment for the local platoon control algorithms is a reactive multi-agent system (RMAS). Reactive agents are relatively simple entities that behave based on their own local perceptions 3 . The reactive approach is one of the most interesting due to its robustness, adaptability and simplicity.

The behavior of each vehicle agent is determined from a physics inspired model that minimizes interactions: each vehicle relates only with the preceding one in the platoon. Steady platoon motion over arbitrary trajectories emerges as a global result of individual behaviors. Statechart of Fig. 2 is a high level representation of vehicle agent behavior. The global behavior integrates two

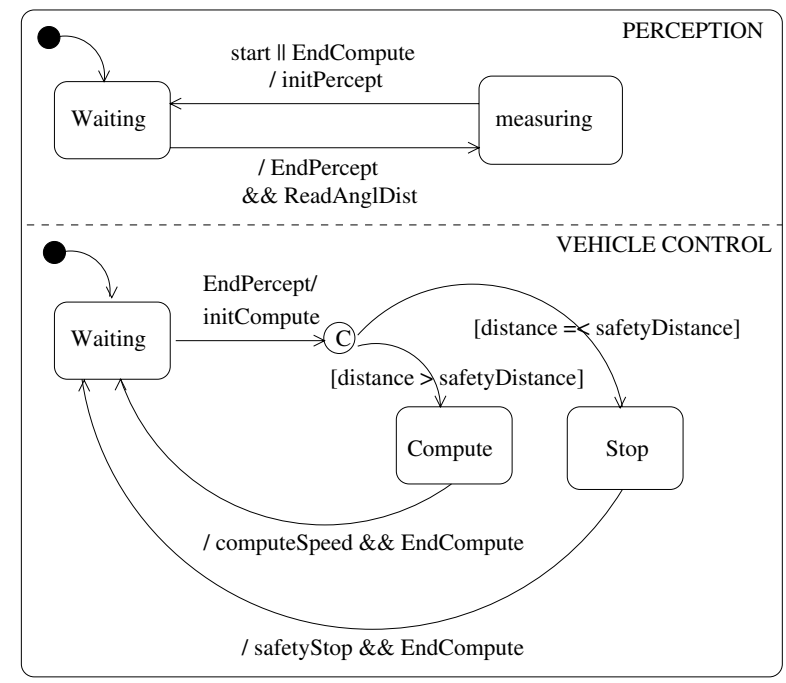

Fig. 2. Inserted vehicle agent behavior 
parallels sub behaviors one corresponding to the perception and the other to the action. The vehicle perception behavior correspond to the measure of the intervehicle vectorial distance using a sensor such as a laser range finder. When the perception cycle is ended, the vehicle control behavior starts. The A distinction is made between the inter-vehicle distance being under the safety distance or not. The safety distance represents the distance required to perform a safety stop. If the inter-vehicle distance is under the safety distance, function safetyStop is called, which triggers a safety stop operation. If the distance is above the safety distance, the function computeSpeed is called to compute the new vehicle's speed reference from the physics inspired interaction model.

\subsection{Interaction Model}

Physics inspired interaction model is used to specify the response of an agent, as determined by its perceptions. The model proposed in this paper is based on three virtual forces: spring force $\boldsymbol{F}_{s}$, damping force $\boldsymbol{F}_{d}$ and surface friction force $\boldsymbol{F}_{f}$, as in [5]6], augmented by torsion force $\boldsymbol{F}_{l}$, introduced to improve the interaction model in curved trajectories, by adding a flexion force to the impedance control model. The virtual link forces are calculated from a classical spring damper model with stiffness $k$, damping $h$ and spring's un stretched length $l_{0}$. Each vehicle $i$ is represented by its position $\boldsymbol{X}_{i}=\left[x_{i}, y_{i}\right]$. The mass of the vehicle is denoted as $m$ (we assume that each vehicle has the same mass). The distance between vehicles is $d=\left\|\boldsymbol{X}_{n+1}-\boldsymbol{X}_{n}\right\|$.

Longitudinal and lateral control references can be determined from just these three forces $\left(\boldsymbol{F}_{s}, \boldsymbol{F}_{d}, \boldsymbol{F}_{f}\right)$, by calculating a new acceleration value. However, simulations and experimentations [6] have exhibited an increase in trajectory error from one vehicle to its follower. This error is due to limitations of the linear impedance control model (Cf. Fig. 3 left). Figure 3 shows the acceleration anticipation and its consequence, trajectory error. To avoid this problem, we propose to add a force, due to the flexing of the impedance control. This force counters the acceleration anticipation. This force is deduced from a virtual moment, result of a flexing spring Moment $=k_{m} * \theta * \boldsymbol{z}$ with $k_{m}$ the torsion spring parameter and $\theta$ the spring angle of flection. This force is computed as
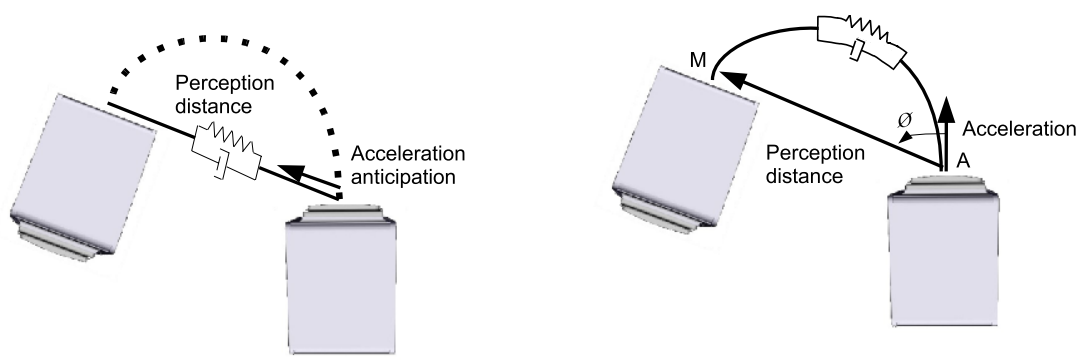

Fig. 3. Acceleration problem and issue 
the vector product of the distance between the moment application point and the preceding vehicle position (C.f Fig. 3 right).

Those forces allow to compute the vehicle's acceleration, by using Newton's law of motion determined relatively to the preceding vehicle reference:

\begin{tabular}{|c|c|}
\hline Spring force & $\boldsymbol{F}_{s}=-k\left(\left\|\boldsymbol{X}_{n+1}-\boldsymbol{X}_{n}\right\|-l_{0}\right) \boldsymbol{u}_{\boldsymbol{n}+\mathbf{1} n}$ \\
\hline Damping force & $\boldsymbol{F}_{d}=-h\left(\dot{\boldsymbol{X}}_{n+1}-\dot{\boldsymbol{X}}_{n}\right)$ \\
\hline Friction force of the surface & $\boldsymbol{F}_{f}=-\lambda \dot{\boldsymbol{X}}_{n}$ \\
\hline Torsion force & $\boldsymbol{F}_{\text {torsion }}=\boldsymbol{M} \boldsymbol{A} \wedge \boldsymbol{M} \boldsymbol{M} \boldsymbol{m e n t}$ \\
\hline
\end{tabular}

Acceleration value:

$m * \boldsymbol{\gamma}=-k\left(\left\|\boldsymbol{X}_{n+1}-\boldsymbol{X}_{n}\right\|-l_{0}\right) \boldsymbol{u}_{n+1}{ }_{n}-h\left(\dot{\boldsymbol{X}}_{n+1}-\dot{\boldsymbol{X}}_{n}\right)+-\lambda \dot{\boldsymbol{X}}_{n}+\boldsymbol{M} \boldsymbol{A} \wedge \boldsymbol{M o m e n t}$

By discrete integration, we can then determine speed and vehicle state (position and orientation). Then the command reference can be computed. In our case, it consists in vehicle direction and speed 11. Model parameters are determined by considering the vehicle's characteristics and constraints.

\section{Inter-Vehicle Distance Stability Analysis}

Analysis of the interaction model by applying classical laws allows to prove that the interaction model satisfies a set of platoon stability properties. The next section presents two kinds of proofs: one applying a direct approach and the other using an energetic point of view.

\subsection{Platoon Stability Based on Transfer Function}

Platoon stability is treated following the "string stability" problem which has been studied in 1977 by Caudill and Garrard [8]. A platoon is said to be stable if, under no other excitations, the error magnitude decreases as it propagates along the vehicle stream. Here, we proposed to prove the string stability relatively to longitudinal control. Formally speaking, if the transfer function of the system composed of two successive vehicles exhibits a magnitude less or equal to 1 , string stability is obtained [21 22]. In order to verify this property, the control law of vehicles $i$ and $i-1$ must be expressed with $\theta=0$ (Longitudinal control): let us suppose A equal to $\frac{\boldsymbol{X}(s)_{i}}{\boldsymbol{X}(s)_{i-1}}$. From laws of motion applied to vehicles $i$ and $i-1$, we obtain

$$
\left\{\begin{array}{l}
m * \ddot{\boldsymbol{X}}_{i}=-k \boldsymbol{X}_{i}-h \dot{\boldsymbol{X}}_{i} \\
m * \boldsymbol{X}_{i-1}=-k \boldsymbol{X}_{i-1}-h \dot{\boldsymbol{X}}_{i-1}
\end{array}\right.
$$

From these equations $A$ can be deduced: $A=\frac{h * s+k}{s^{2}+h * s+k}$.

String stability is guaranteed only if: $|A(j \omega)| \leq 1$. This condition is verified with $k \leq \frac{m * \omega^{2}}{2}$. This study proof that our system is stable to any frequency. The error propagation will be attenuated through the platoon.

${ }^{1}$ The choice of a command law takes into account the characteristics of the test vehicle used in our laboratory. 


\subsection{Inter-Vehicle Distance Stability Proof Based on Energy}

We intend to check if the stability 2 of the system is kept in run time. To this end we apply Lyapunov stability of motion conditions [720. The system energy results from the addition of kinetic and potential energies. We take vehicle $i$ as reference frame to express the energy.

$$
\begin{aligned}
& E=E_{\text {kinetics }}+E_{\text {potential }}=E_{\text {kinetics }}+E_{\text {pot }}\left(F_{s}\right)+E_{\text {pot }}\left(F_{\text {torsion }}\right) \\
& E=\frac{1}{2} * m *(\dot{\boldsymbol{X}} \cdot \dot{\boldsymbol{X}})+\frac{1}{2} * k *(\boldsymbol{X} . \boldsymbol{X})+\int_{X} \int_{\theta} \boldsymbol{F} . d Y d \theta
\end{aligned}
$$

By applying Lyapunov stability of motion conditions [7|20, we obtain

$$
\left[\begin{array}{l}
\frac{\partial E(x, y, \theta)}{\partial x} \\
\frac{\partial E(x, y, \theta)}{\partial y}
\end{array}\right]=\left[\begin{array}{l}
x_{2}^{2}\left(k_{m} \sin (\theta)-h\right) \\
y_{2}^{2}\left(k_{m} \cos (\theta)-h\right)
\end{array}\right]
$$

The derivative of Energy is negative if the angular $h * k_{m}$ is greater than 1 since $\theta \in[-\pi / 2, \pi / 2]$. Applying Lyapunov stability of motion conditions as in 6]4, shows that the system is stable when time tends to infinity. Thus, the distance between vehicles tends to the un stretched spring length when the platoon moves without environmental influence, if the condition on rotation angle $(\theta \in[-\pi / 2, \pi / 2])$ is verified.

\section{Experimentation Results}

The physics inspired interaction model previously defined was the base to the specification of the local platooning control algorithms. Based on those algorithms, simulation experiments were designed and performed to check a set of safety platoon conditions. The simulations have been made on a simulator able to take into account vehicle dynamics properties. The simulator has 3D graphic display capabilities developed with OpenSceneGraph 3 , in connection with a dynamics enginet used to model the physical aspects (realistic model of a car moving in a material environment.) The vehicle dynamics have been modeled as a physical box with 4 shock absorber wheels. Vehicle models integrate the dynamic properties of a real vehicle. Simulations have been realized in order to visualize inter-vehicle distance and vehicle position evolution within a platoon (C.f Fig. (4).

In order to perceive the environment and the preceding vehicle, the model of a laser range finder has been included in the follower vehicles. The laser measurement system is based on a time-of-flight measurement principle. Simulations have been performed to verify the platoon evolution during startup and lateral displacement situations.

${ }^{2}$ platoon stability is represented by the stabilization of the distance between each follower vehicle.

3 http://www .openscenegraph.org/projects/osg

4 http://www .ode.org/ 


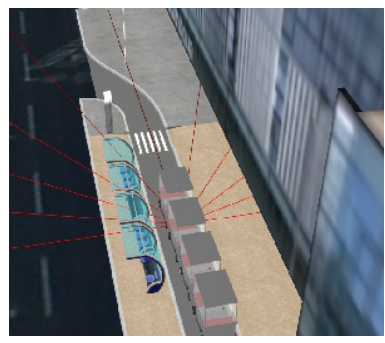

Fig. 4. 3 Dimensions simulator: platoon of 5 vehicles into a station (rays correspond to the second vehicle laser range finder)

\subsection{Platoon Startup}

The simulation starts with 4 platoon vehicles at 1.6 meter of inter-vehicle distance.

The first vehicle is controlled by the user. Each follower vehicle follows the preceding one, by executing its own perception-based behavior. Figure 5 shows the inter-vehicle distance during the platoon evolution. The inter-vehicle distance value is initialized to 1.6 meter with a vehicle speed of $30 \mathrm{~km} / \mathrm{h}$ then a safety stop is performed. All inter-vehicle distances decrease to 0.5 meter. This shows that there are no collisions but only some little oscillations in simulations.

\subsection{Platoon Lateral Displacement}

Platoon lateral displacement simulation (station entering and exiting) have been realized to check that each vehicle follows correctly its predecessor.

Figures 6]illustrates platoon evolution during station exiting, by tracing both the leader and follower trajectories. This figure exhibits a little increase in

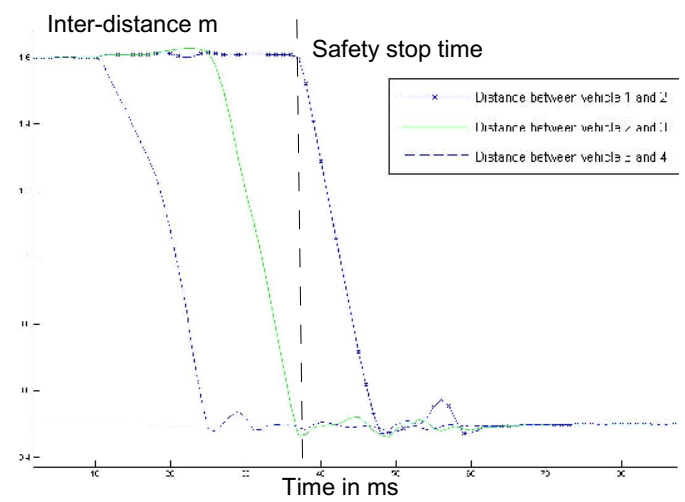

Fig. 5. 3D simulator: inter-vehicle distance evolution 


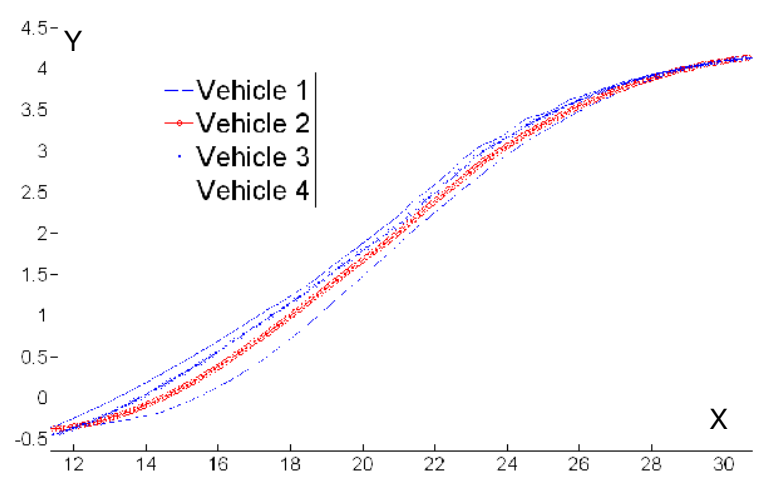

Fig. 6. 3D simulator: Trajectory error during the station exit

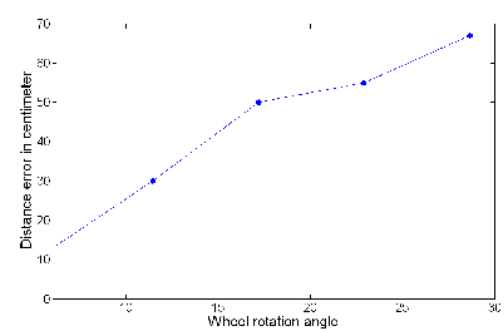

\begin{tabular}{|c|c|c|c|}
\hline $\begin{array}{c}\text { Wheel rotation } \\
\text { (degree) }\end{array}$ & $\begin{array}{c}\text { Curve } \\
\text { radius }\end{array}$ & $\begin{array}{c}\text { medium } \\
\text { error }\end{array}$ & $\begin{array}{c}\text { maximum } \\
\text { error }\end{array}$ \\
\hline 5.73 & $18 \mathrm{~m}$ & $12 \mathrm{~cm}$ & $14 \mathrm{~cm}$ \\
\hline 11.46 & $9 \mathrm{~m}$ & $30 \mathrm{~cm}$ & $40 \mathrm{~cm}$ \\
\hline 17.2 & $6 \mathrm{~m}$ & $50 \mathrm{~cm}$ & $65 \mathrm{~cm}$ \\
\hline 22.9 & $4.5 \mathrm{~m}$ & $55 \mathrm{~cm}$ & $67 \mathrm{~cm}$ \\
\hline 28.65 & $3.6 \mathrm{~m}$ & $67 \mathrm{~cm}$ & $75 \mathrm{~cm}$ \\
\hline
\end{tabular}

Fig. 7. 3D simulator: trajectory error in curve

trajectory error from one vehicle to its follower. The maximum of this error does not exceed the width of a wheel (C.f Fig. 6 left).

\subsection{Relationship between the Curve and the Trajectory Error}

The simulation presented next was intended to show the relationship between the bend radius and the trajectory error as presented previously.

This simulation corresponds to the vehicle starting with a given wheel angle. Then, the simulator computes the evolution of the distance error. Figures 7 and table illustrates the error in the curve. This error increases with the magnitude of the wheel angle.

\section{Conclusion}

The aim of this article was to present a better local platoon control based on the bending of a virtual spring damper. Platoon control, as presented in this paper, bases only on local perception capabilities. Each vehicle behavior is deduced from a physics inspired interaction model an embodied in a reactive agent architecture. The use of physics inspired forces enables an easier tuning of the interaction model parameters and the adaptation to any kind of vehicle. Besides, the physic 
model has been used to prove platoon stability, by using classic physical proof method: energy analysis. Analogously, another stability proof have been realized following a transfer-function approach. To assert the transition from abstract to concrete, simulations have been implemented to show the applicability of the theoretical model. Some simulations have been made on a $3 \mathrm{D}$ simulator which integrates vehicle dynamic properties (maximal speed and acceleration, ....). These experimentations exhibit a little curved trajectory error during the platoon evolution and indicate that the presented approach improves platoon quality. We are now working on the application of this model on real vehicles. Moreover, we are also advancing into further research on the use of Formal Models in order to prove some application properties and to ensure a zero default embedded software for real vehicles.

\section{References}

1. Hedrick, J.K., Tomizuka, M., Varaiya, P.: Control issues in automated highway systems. IEEE Control Systems Magazine, 21-32 (1994)

2. Debojyoti, M., Asis, M.: Pollution control by reduction of drag on cars and buses through platooning. Int. J. of Environment and Pollution 30(1), 90-96 (2007)

3. Ferber, J.: Multi-Agent System: An Introduction to Distributed Artificial Intelligence. Addison Wesley Longman, Harlow (1999)

4. Gaud, N., Gechter, F., Galland, S., Koukam, A.: Holonic multiagent multilevel simulation: Application to real-time pedestrians simulation in urban environment. In: Twentieth International Joint Conference on Artificial Intelligence, IJCAI 2007, pp. $1275-1280$ (2007)

5. Contet, J.M., Gechter, F., Gruer, P., Koukam, A.: Physics inspired multiagent model for vehicle platooning. In: International Conference on Autonomous Agents and Multiagent Systems AAMAS (2007)

6. Contet, J.M., Gechter, F., Gruer, P., Koukam, A.: Multiagent System Model for Vehicle Platooning with Merge and Split Capabilities. In: Third International Conference on Autonomous Robots and Agents, pp. 41-46 (2006)

7. Taylor, F., Lyapunov, A.M.: The general problem of the stability of the motion (1992)

8. Garrard, W.L., Caudill, R.J.: Dynamic Behavior of Strings of Automated Transit Vehicles. SAE Transactions, 1365-1378 (1977)

9. Martinet, P., Thuilot, B., Bom, J.: Autonomous Navigation and Platooning using a Sensory Memory. In: International IEEE Conference on Intelligent Robots and Systems, IROS 2006, Beijing, China (2006)

10. Myung, J.W., Jae Weon, C.: A relative navigation system for vehicle platooning. In: SICE 2001, Proceedings of the 40th SICE Annual Conference, pp. 28-31 (2001)

11. Fritz, H.: Longitudinal and lateral control of heavy duty trucks for automated vehicle following in mixed traffic: Experimental results from the CHAUFFEUR project. In: IEEE Conference on Control Applications - Proceedings, vol. 2, pp. 1348-1352 (1999)

12. Ioannou, P., Xu, Z.: Throttle and brake control systems for automatic vehicle following. IVHS Journal, 345 (1994)

13. Moskwa, J.J., Hedrick, K.J.: Nonlinear algorithms for automotive engine control. IEEE Control Systems Magazine 10, 88-93 (1990) 
14. Daviet, P., Parent, M.: Longitudinal and lateral servoing of vehicles in a platoon. In: Proceedings of IEEE Intelligent Vehicles Symposium, pp. 41-46 (1996)

15. Sheikholeslam, S., Desoer, C.A.: Longitudinal control of a platoon of vehicles with no communication of lead vehicle information: A system level study. IEEE Transactions on Vehicular Technology 42, 546-554 (1993)

16. Lee, H., Tomizuka, M.: Adaptive vehicle traction force control for intelligent vehicle highway systems (IVHSs). IEEE Transactions on Industrial Electronics 50, 37-47 (2003)

17. Kehtarnavaz, N., Griswold, N.C., Lee, J.S.: Visual control of an autonomous vehicle (BART)-The vehicle-following problem. IEEE Transactions on Vehicular Technology 40, 654-662 (1991)

18. Gehrig, S.K., Stein, F.J.: Elastic bands to enhance vehicle following. In: IEEE Conference on Intelligent Transportation Systems, Proceedings, ITSC, pp. 597$602(2001)$

19. Yi, S.-Y., Chong, K.-T.: Impedance control for a vehicle platoon system, Mechatronics (UK), vol. 15, p. 627 (2005)

20. Leipholz, H.: An introduction to the stability of Dynamic systems and rigid bodies (1987)

21. Swaroop, D., Hedrick, J.K.: String stability of interconnected systems. IEEE Transactions on Automatic Control 41, 349-357 (1996)

22. Liang, C., Peng, H.: Optimal Adaptive Cruise Control with Guaranteed String Stability (1998)

23. Klancar, G., Matko, D., Blazic, S.: Wheeled Mobile Robots Control in a Linear Platoon. Journal of Intelligent and Robotic System (2008) 